\title{
Serial properties of behavior and their chemical modification
}

\author{
BERNARD WEISS, JOHN M. ZIRIAX, and M. CHRISTOPHER NEWLAND \\ University of Rochester, Rochester, New York
}

\begin{abstract}
Certain features of operant behavior that are sensitive to the adverse effects of chemical exposure can be obscured by exclusive experimental reliance on global measures such as response rate. Temporal patterning of behavior is the clearest example. Reinforcement schedules studied in behavioral pharmacology and toxicology reveal novel consequences of chemical treatment when subjected to analyses of their temporal and serial properties. Fixed-ratio performance, for example, undergoes not only changes in response rate, but also displays distinctive shifts in interresponse time patterning, changes in the interresponse time distribution, and deterioration in what might be termed the cohesiveness of the ratio. Variable-interval performance also may change in distinctive ways that produce altered response patterning without marked changes in rate. Sequential dependencies, as measured by techniques of time series analysis, may also reveal effects not reflected by response rates. Spaced responding and autoregressive schedules provide examples. The serial and temporal properties alluded to above can be described and analyzed by a variety of quantitative techniques that also yield information of theoretical interest.
\end{abstract}

Behavior is a process, not a state. Yet although this principle is generally acknowledged, it is often ignored: the process is condensed into an average such as rate. This practice obscures some of the most important and intriguing facets of behavior: its sequential properties. Surely, if early events did not influence later ones, a science of behavior would not exist. Previous symposia in this series, in fact, have aroused debates, such as those on maximizing and matching, that have eddied around minute details of process. To add biological and pharmacological variables only magnifies such issues. In the present paper, we plan to feature one of these: serial or sequential relationships and what they add to an experimental analysis. Two kinds of situations will be examined, one of which makes sequential dependencies an explicit element in a contingency, while the other induces such effects indirectly. Those aspects that clarify the actions of chemical agents will be stressed here.

Behavioral pharmacology and toxicology depend upon behavioral science to provide a technology suitable for assessing chemical influences. When that technology proves inadequate, it hampers not only the ability to interpret the consequences of chemical treatment, but even the ability to design the appropriate experiments. In one major respect, that technology is inadequate. Especially during the past two decades, when such impressive

Presented at the Seventh Harvard Symposium on Quantitative Analyses of Behavior: Biological Determinants, Harvard University, June 8-9, 1984. Preparation of this review was supported in part by Grants MH-11752 (National Institute of Mental Health), ES-01247, ES-01248, and ES-30354 (National Institute of Environmental Health Sciences), and by a grant from the U.S. Department of Energy. Correspondence may be addressed to Bernard Weiss, Environmental Health Sciences Center, University of Rochester, Medical Center, Rochester, NY 14642. progress has been achieved in understanding the molecular processes governing the chemistry of the nervous system, specialists in the experimental analysis of behavior seem to have turned more and more to the kind of gross phenomena that can be conveniently accommodated by mathematical models such as the matching law. This paper is not the place to review such a complex issue, but the tendency to ignore what are called molecular behavioral processes in favor of familiar molar ones such as rate has surely exercised some influence on the choice of endpoints made by those who study the joint actions of chemicals and behavior.

An examination of molecular influences on behavior requires direct examination of both the microstructure of behavior and the molecular contingencies acting up on it. Theoretical and quantitative accounts of behavior at the molecular level, therefore, should emphasize moment-tomoment variations in both behavior and the reinforcement contingencies. A theoretical account that includes ratio size, reinforcement magnitude, and effort, but ignores molecular aspects of behavior, will not predict the molecular regularities that appear in behavior. Theoretical efforts like Mazur's (1982), although perhaps useful in describing gross, molar features of behavior, reveal little about how such molar structure develops, because they ignore the molecular contingencies and fail to predict microstructure.

The aspect of behavior most commonly ignored by molar analyses, and the lack most often stressed by their critics, is its serial patterning. Coercing the stream of behavioral events into a summary measure such as rate bypasses the labor of dissecting the microstructure of behavior, and affords the experimenter, as noted by Silberberg, Hamilton, Ziriax, and Casey (1978), a surface simplicity and an ease of recording. To ignore serial 
patterning, however, means to assume that responses occurring in time are independent of each other. Many writers have protested the fallacy of such an assumption. Miller and Frick (1949), even with the crude technology then available, were able to demonstrate serial dependencies in a prototypical operant situation. Further examples have been offered by Millenson and Hurwitz (1961), Schwartz (1980), Silberberg and Ziriax (1982), Williams (1968), and others. These examples come largely from situations in which explicit requirements for serial patterning were absent. A recent analysis of sequential dependencies has revealed the type of structure found even in fixed-interval (FI) schedules. Although cumulative records suggest that response rates accelerate as the interval progresses, an analysis of sequential interresponse times (IRTs) showed something quite different (Gentry, Weiss, \& Laties, 1983). Instead of a progressive decrease in IRTs, which would be expected if the response rate accelerated, the apparent scallop was shown to arise from a progressive decrease in the frequency of pauses that separate bursts of responding. That is, as the interval progresses, the pauses or long IRTs decline in frequency, while the bursts remain unchanged. The FI scallop may be an artifact of a visual or mechanical averaging of the lines produced by cumulative records or by averaging counters over a session (Branch \& Gollub, 1974). Even more extended sequences were documented, such as a small negative correlation between the number of responses emitted during successive intervals. Such phenomena suggest that molecular contingencies operate whether explicitly programmed by the experimenter or not. To the extent that behavior is controlled as a molecular process, a molecular analysis must be an integral part of an experimental analysis of behavior.

It is no surprise that distinctive sequential patterns arise without explicit contingencies, given the ease with which they can be shaped and maintained by specific contingencies. Shimp $(1973,1981)$ has demonstrated such patterning in several contexts, including positional and IRT sequences. Shimp (1973) noted the "widespread neglect of sequential phenomena"' in the literature on matching, and he has been a vigorous advocate for their inclusion in any attempts to account for choice behavior. As long ago as 1956, Morse and Herrnstein demonstrated that specified IRT sequences preceding reinforcer delivery responded differentially to methamphetamine and pentobarbital.

\section{INDIRECT DEPENDENCIES}

Some of the contingencies evoking significant serial relationships have long traditions in behavioral pharmacology. These may be defined as indirect dependencies, because they are not explicitly imposed by the experimenter. Instead, they arise adventitiously from the way in which certain patterns of responding are molded by the schedule of reinforcer delivery. Skinner was quite aware of this aspect of schedule-controlled behavior; in The Behavior of Organisms (1938), he noted that schedules were a way of arranging the occurrence of certain patterns of behavior at the moment of reinforcement. In the present section of this paper, we will discuss three traditional schedules (spaced-responding or DRL, fixedratio, and variable-interval); we will then present examples of how drug treatments may modify the characteristic serial patterning of responding associated with each.

\section{Spaced Responding}

Spaced-responding performance, or differential reinforcement of low-rate (DRL) performance, is maintained by requiring a minimum (sometimes coupled with a maximum) interval between successive responses. Typically, the minimum is relatively long, so that the subject eventually learns to engage in other behaviors during the interval. Sometimes, these behaviors become overtly detectable, especially if the experimenter provides an explicit alternative response (Laties, Weiss, \& Weiss, 1969). Although such interpolated behaviors often seem rather inconsistent, it may be possible to detect some underlying unity in the process under certain conditions, as when the interpolated behaviors are captured by a measurement device.

One experiment was performed with 3 monkeys maintained in primate chairs during experimental sessions. The monkeys responded by pressing telegraph keys with attached knobs. Reinforcement consisted of several drops of fruit drink delivered through a spout. An extensive description of the acquisition history appeared in Weiss (1970b). Since all IRTs were preserved in real time by an on-line computer that also controlled the experiment, it was possible to examine the serial relationships in great detail and in many different ways, as described below.

(1) As spaced-responding performance developed, short IRTs dropped out, and the peak of the IRT distribution began to move toward the minimum interval (here, $20 \mathrm{sec}$ ) required for reinforcement. The clearest index of the shift in performance was the dwell time, computed as the proportion of session time occupied by IRTs of various length.

(2) Plots of all IRTs, when compared to plots of only IRTs following reinforcement and to plots of IRTs separated from reinforcement by one or more interposed IRTs, indicated an increased proportion of longer IRTs following reinforcement. That is, reinforced IRTs tended to be followed by other reinforced IRTs. Early in training, this process was visible in cumulative records as trains of reinforced responses (Figure 1). Even after extensive experience with the schedule, at a stage when most IRTs were long enough to secure reinforcement, a similar pattern was visible as alternating trains of longer and shorter IRTs.

(3) Such serial dependencies may be quantified in various ways. The joint interval histogram plots IRT $_{i}$ against $\mathrm{IRT}_{i+n}$. With $n=1$, the joint-interval histogram typically reveals an ellipse that corresponds to a positive correlation between the lengths of successive IRTs. More advanced techniques of time-series analysis can also help one describe and understand the process. Autocorrelograms and power spectra are widely used statistical tools 

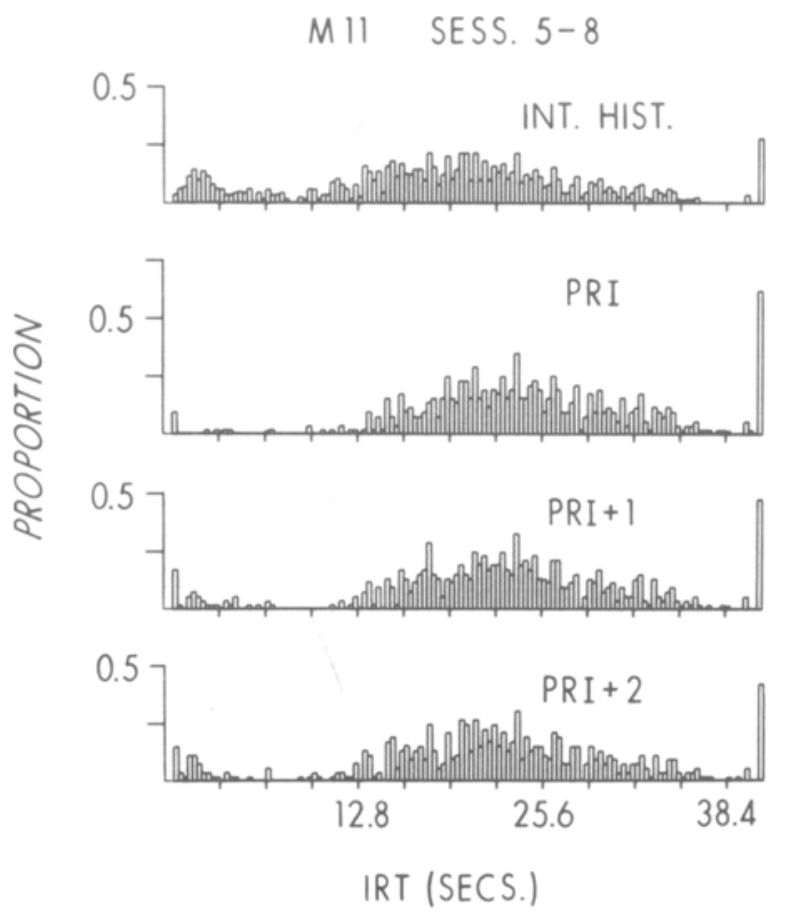

Figure 1. Histograms of IRTs on DRL $20 \mathrm{sec}$ for Monkey M11, based on Sessions 5-8. The histogram at the top of the figure includes all IRTs during these sessions. The histogram labeled PRI includes only IRTs following a reinforced response. Those labeled PRI +1 and PRI + 2 represent IRTs displaced by 1 and 2 intervening responses following a reinforced response.

for this purpose. The autocorrelogram describes the serial dependencies as a sequence of correlation coefficients of

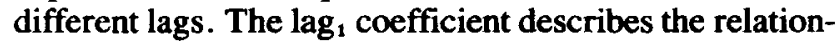
ship between the original time series and the same time series displaced by one time interval. The $\operatorname{lag}_{2}$ coefficient correlates the original time series with the same time series displaced by two time intervals; and so on. Periodicities in the autocorrelogram tend to repeat, which makes its statistical properties somewhat obscure. A spectral analysis converts the autocorrelation data into a more tractable statistical form. It breaks down the total variance of the time series (after subtracting the mean) into components attributable to fluctuations of different length. These fluctuations, like those of a time-varying electrical signal, are then expressed in terms of frequency, and the associated variance is expressed as power. A time series with slow, long-wavelength fluctuations will then show most of its power at low frequencies, and a rapidly varying time series will show a preponderance of power at high frequencies. Such an analysis was undertaken by Weiss, Laties, Siegel, and Goldstein (1966), to quantify some of the serial aspects of spaced-responding performance. It demonstrated that, at least under the specific conditions of the experiment, spaced-responding performance displays long-wavelength oscillations in IRT duration.

The character of the serial dependency, not just response rate or IRT duration, becomes modified by drugs (Weiss \& Laties, 1967). In 1 monkey performing under a DRL 20 -sec contingency, a dose of $0.2 \mathrm{mg} / \mathrm{kg} d, l$-amphetamine sulfate affected the response rate minimally, but it increased variability or total power and clearly enhanced serial correlation. This effect can be grasped by inspecting Figure 2, a joint-interval histogram plotting the relationship between successive IRTs. Note the elongation of the distribution after the drug was administered. As on scatterplots in general, such a pattern indicates a higher correlation coefficient. Such an effect is not a universal property of this class of drugs; contingencies naturally play an overwhelming role in determining the character of the response. The spaced-responding contingency, if it is to gain control of performance, must shape some kind of intervening behavior. It seems unlikely, moreover, that such behavior is random-that is, unrelated to the contingency. If amphetamine reduces the consistency of the intervening behaviors, the IRTs will display less homogeneity. Such enhanced variability could lead to the increase in slow fluctuations of IRTs shown by the powerspectrum calculations (Weiss \& Laties, 1967).
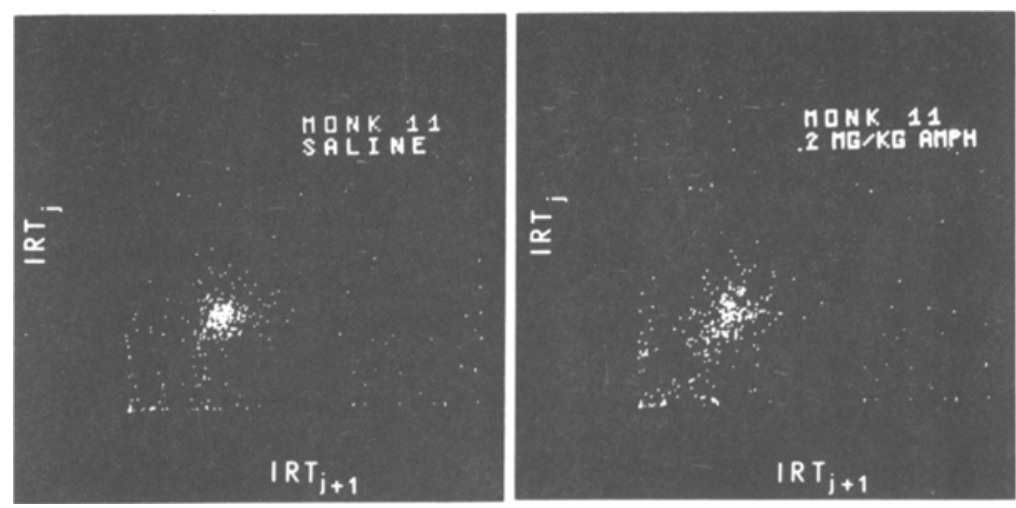

Figure 2. Joint interval histograms comparing DRL-20 performance after saline administration to performance after administration of $0.2 \mathrm{mg} / \mathrm{kg} d, l$-amphetamine sulfate. 


\section{Fixed Ratio}

Ratio performance tends to be so robust that it almost has achieved the status of a control procedure, serving as a baseline against which to measure nonspecific effects such as physical impairment or gastrointestinal illness. Its stability is, however, partly an illusion that arises from the failure to inspect its microproperties. This is apparent from an experiment with pigeons trained on fixed-ratio schedules, in which recording the behavior in real time permitted a close examination of the acquisition process.

Gott and Weiss (1972) traced the development of mature ratio performance in several different ways, beginning with the transition from continuous reinforcement to FR (fixed-ratio) 30. As Ferster and Skinner (1957) first demonstrated, the transition process is ragged. Long pauses are interpolated between variable bursts of responses. With experience, the performance takes on a rather uniform character, consisting of a long pause at the beginning of the ratio followed by a fairly cohesive train of responses, emitted at high rates, until reinforcement. The longer IRTs, which Gott and Weiss termed outliers, and which were defined as lasting $2 \mathrm{sec}$ or more, occurred about equally at all ordinal IRT positions beyond the first two or three during the intermediate phases of acquisition. As they dropped out, the performance became more and more cohesive, as pecks were emitted with a fairly regular rhythm.

Figure 3 shows mean ordinal IRTs, from one FR drug session and corresponding control days, produced by an experienced pigeon (Weiss \& Gott, 1972). After the first few IRTs, the intervals were relatively short. But there was a definite pattern in the ordinal control plots (left and right panels), with IRTs in the middle positions tending to be shorter than those on the end. Ordinal patterns tend to be unique to each subject, even though they also drift over time to assume somewhat different profiles. The middle panel is an equivalent ordinal plot from a session

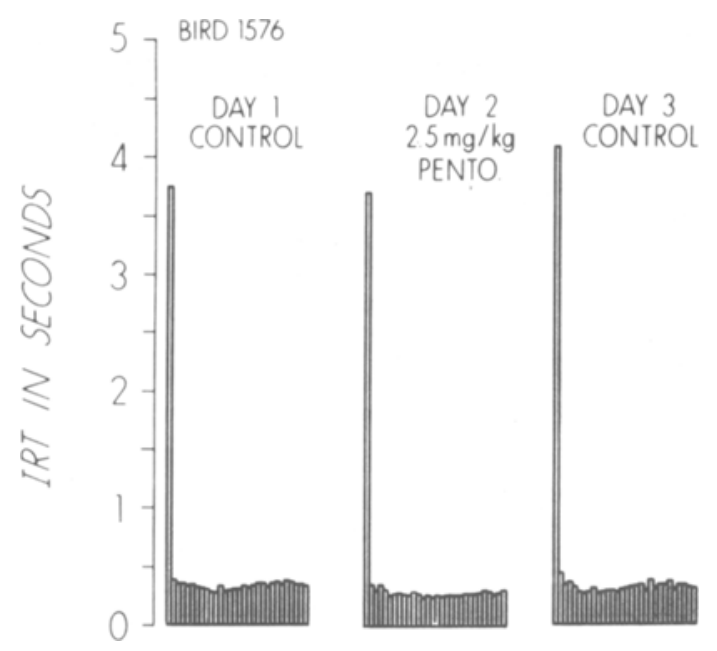

Figure 3. Mean ordinal IRTs from three successive fixed-ratio 30 sessions. $2.5 \mathrm{mg} / \mathrm{kg}$ pentobarbital was administered to the pigeon before the second session.
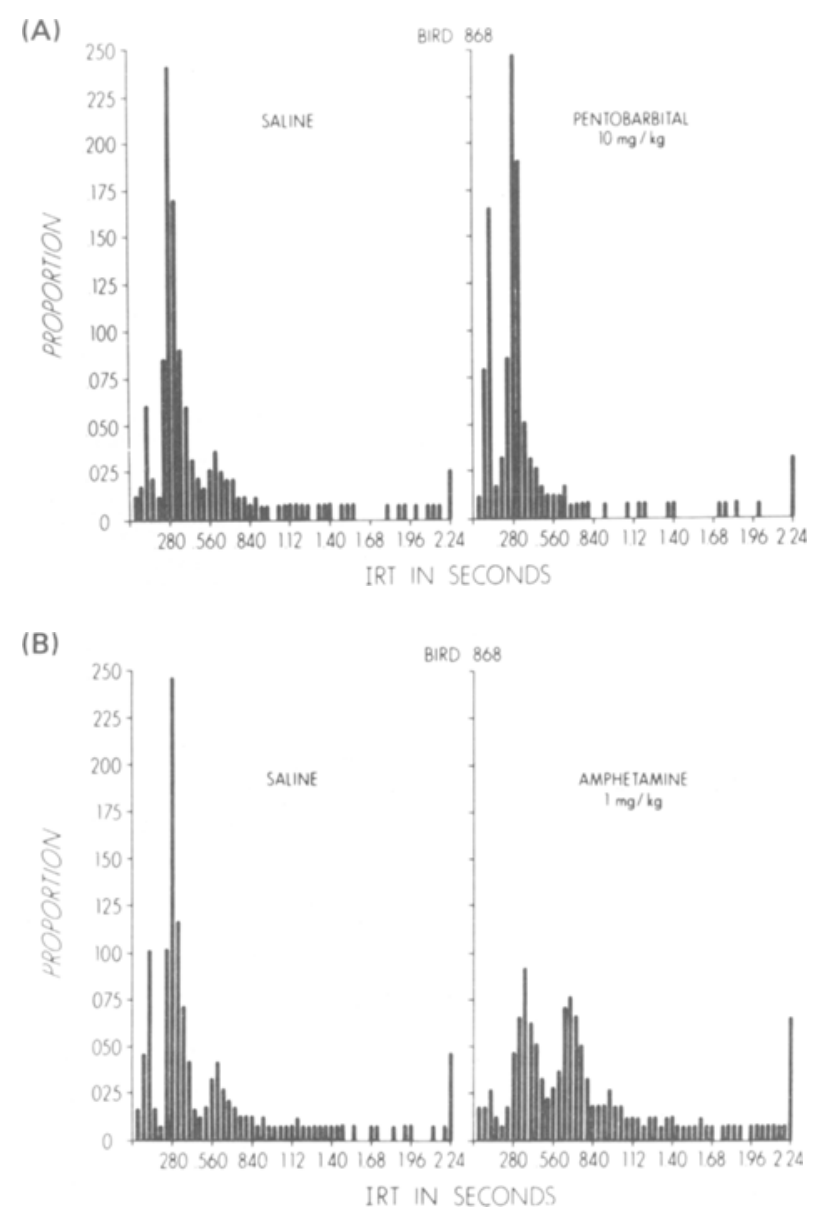

Figure 4. (A) IRT distributions, based on 40-msec bins, derived from fixed-ratio 30 performance in a pigeon. Performance after saline is compared with performance after sodium pentobarbital. (B) Equivalent plot for amphetamine.

preceded by the injection of $2.5 \mathrm{mg} / \mathrm{kg}$ sodium pentobarbital. Several characteristics of the performance changed with drug treatment. First, note that the mean IRTs, at all positions within the ratio, were shortened after drug administration. Second, note that the ordinal FR pattern changed. It became flatter (i.e., more uniform), and the dip in IRT duration was no longer visible about one third of the way through the ratio.

It is not novel to find elevated FR response rates after barbiturate treatment. But what about their source? Rates typically are calculated simply by dividing total responses by total time. Such a practice gives overwhelming prominence to the long, highly variable pause that follows reinforcement, and it inhibits the search for potential behavioral mechanisms. Suppose, for example, that the source of the ordinal pattern after drug treatment is a change in topography. That is a cogent possibility easy to visualize if the IRT distribution is divided into narrow intervals. Figure 4A compares performance after saline administration with performance, in the following session, after sodium pentobarbital administration. Several proper- 
ties of the distribution changed. First, the dominant mode of intervals in the range of about $300 \mathrm{msec}$ was shifted left. These are pecks that consisted of cleanly delivered strikes at the key. Second, the proportion of very short IRTs increased. Gott and Weiss (1972) termed these "nibbles," because they were produced by rapid beak oscillations, not pecks. Third, the mode at about $600 \mathrm{msec}$ was almost totally eliminated. This "harmonic" mode represents incomplete operants, and almost surely arose from pecks, coordinated rhythmically with the dominant 300 msec mode, that struck the chamber wall, that never reached the key, or that struck the key with insufficient force to interrupt the contacts. Such harmonic modes are especially prominent early in FR training and tend to fade with additional experience on the schedule. Amphetamine produced just the opposite effects, namely, a shift of the primary mode to a longer duration, a reduction in very short IRTs, and an enhancement of harmonics (Figure 4B). It is almost as though amphetamine had resurrected response properties previously extinguished (cf. Skinner, 1938) while pentobarbital had restricted the pigeon's environment to one more narrowly focused on the response key. In fact, the pigeons were observed to stand closer to the key after drug treatment-the only way in which they could increase the proportion of "nibbles."

Complex temporal patterns of the kind described above are important clues to understanding how chemical treatments modify ratio performance. These patterns suggest that shifts in topography contribute significantly to such modifications. But temporal patterning cannot be neglected even in behavioral accounts of ratio performance. The "molecular" model of ratio performance described by Mazur (1982), for example, could be expanded to incorporate the findings of Gott and Weiss (1972) on the distribution of IRTs, especially the intrusion of pauses. Further, the differential response to drugs of the different IRT clusters indicates that molecular accounts probably need to deal with variable topographies (Weiss \& Gott, 1972).

\section{Variable Interval}

VI performance will be discussed only briefly, because it has been discussed in other articles (e.g., Weiss, 1973a, 1981). One of its most distinctive properties, instability, is not merely ignored, but shunned, in molar investigations. The IRT distributions plotted by adequately sensitive time scales reveal a performance with prominent multiple modes, and, moreover, one that shifts continuously, displaying what Morse and Kelleher (1970) termed metastability. Behavior, of course, is a dynamic phenomenon; it is certain to undergo transformations as it connects with experimental contingencies. Chemical treatment will alter rate only as a byproduct of more basic behavioral mechanisms that a microanalysis can sometimes identify.

Figure 5 illustrates such a possibility. The main portion of the upper plot is an IRT distribution from a pigeon maintained on a VI 2.5-min schedule of reinforcement (Weiss, 1973a). Note the dominant mode at about

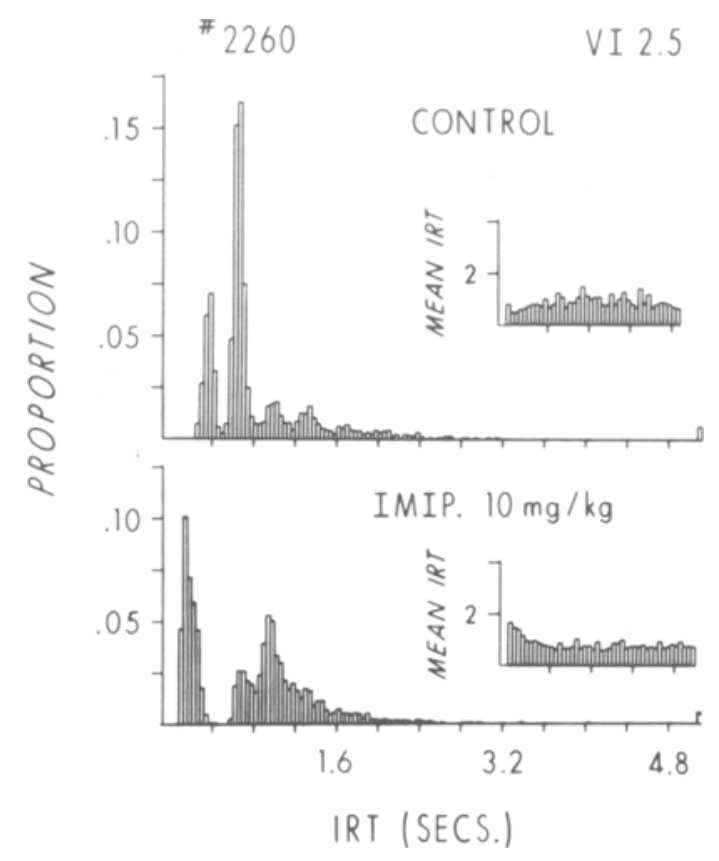

Figure 5. IRT distributions, based on 40-msec bins, on V1 $2.5 \mathrm{~min}$ after saline and after $10 \mathrm{mg} / \mathrm{kg}$ imipramine hydrochloride. Insets trace mean IRTs (in seconds) for each consecutive 100 IRTs during the experimental session; abscissa tick marks are placed every 1,000 IRTs.

$700 \mathrm{msec}$, which seems to be a harmonic of the earlier mode at about half that value-an interpretation based on the two later modes, which are close to multiples of the first peak. The lower plot is an IRT distribution after the administration of $10 \mathrm{mg} / \mathrm{kg}$ imipramine hydrochloride. Observe the position of the first peak, which is about $120 \mathrm{msec}$ less than the first peak of the upper plot. The total profile of the plot is even more sharply different, extending from the gap at about $500 \mathrm{msec}$ to the two apparently unrelated peaks at longer durations. Had only total response rate been recorded, as shown in the insets, we would simply have concluded that imipramine slightly lowers rate. It could not have been seen that such an outcome arose from a pattern of responding in which trains of short IRTs apparently alternated with IRTs about three times as long. Again, the source of the effect was quite possibly a shift in topography.

\section{EXPLICIT DEPENDENCIES}

\section{Autoregressive}

Variability pervades all operant performance. Some contingencies, such as DRL and pacing schedules (Ferster \& Skinner, 1957), reduce variability by setting boundaries to acceptable IRTs. Others, such as fixed-ratio, do so by promoting regular trains of responses. On-line computer control of reinforcement contingencies, however, permits variability (or consistency) to be controlled directly. The earliest attempt to do so relied on a contin- 


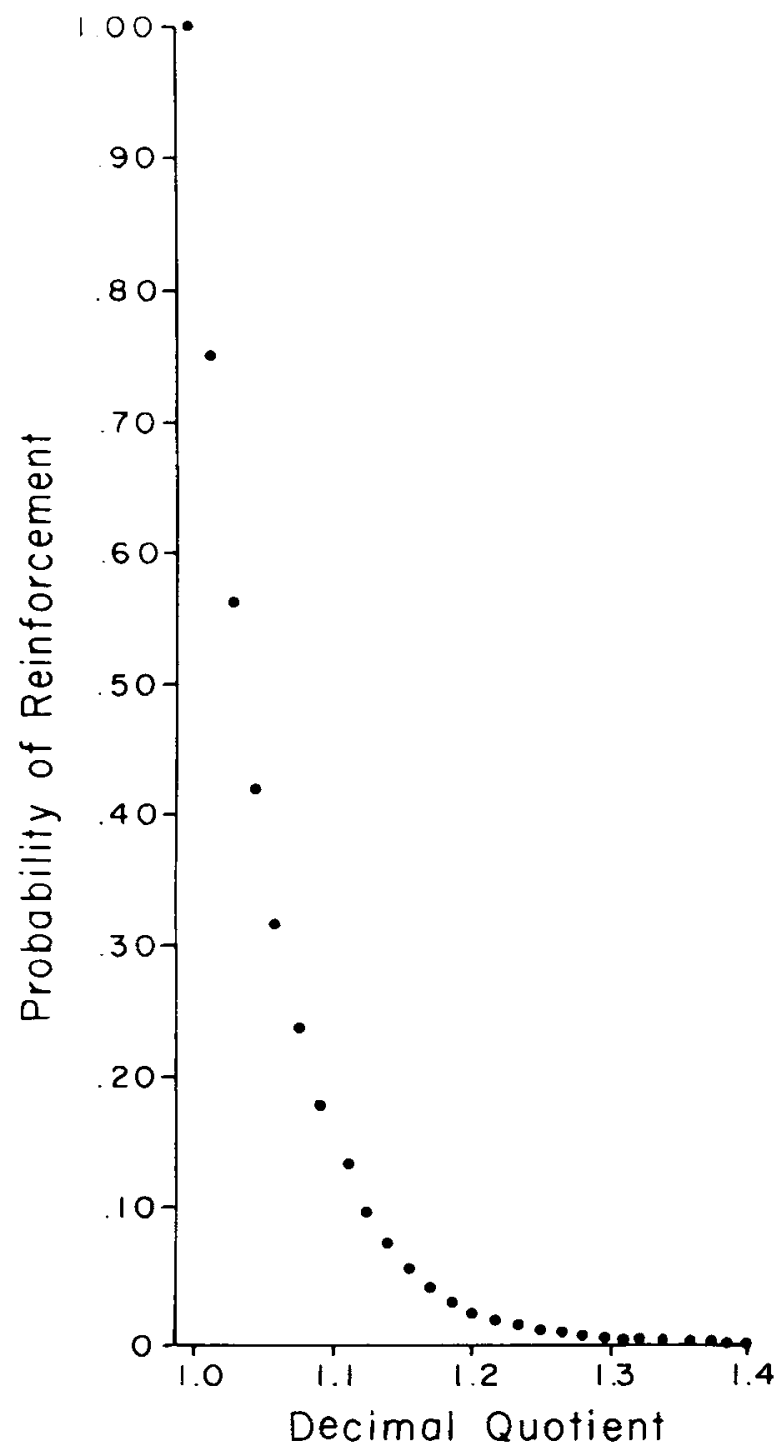

Figure 6. Autoregressive reinforcement schedule (ARG) function relating probability of reinforcement to quotient of $\mathrm{IRT}_{t+1}$ and $\mathrm{IRT}_{t}$, with the larger value placed in the numerator.

gency termed the autoregressive (ARG) reinforcement schedule (Weiss \& Laties, 1965; Weiss, 1973b). Its basis is plotted in Figure 6. After each response, the resultant IRT was compared to the previous IRT by taking the quotient of the two. The closer the quotient to 1.0 , the higher the probability of reinforcement. (The program used the quotient to locate a register in memory. It contained a number that corresponded to the probability that its value would exceed a random number generated from a defined distribution.) This contingency elicited quite narrow IRT distributions from monkeys maintained in a primate chair during testing and given small quantities of fruit drink for successful responses. The response device was a telegraph key.

Gage (1970) studied the effects of drugs on ARG performance. These data are more clearly revealed by expectation-density plots (Poggio \& Viernstein, 1964) than by conventional IRT distributions. Figure 7 shows how such plots are generated. In essence, they provide the equivalent of an autocorrelation function for a time series defined by intervals between unit impulses rather than by signal amplitudes. They can be viewed as a way of sweeping through time to observe the subsequent history of a response. Figure 8 is based on the performance of a cebus monkey given various doses of $d, l$-amphetamine sulfate or sodium pentobarbital. It reveals that increasing doses of amphetamine diminished the already narrow variability even further. In contrast, pentobarbital smeared, so to speak, the prominent peaks of the expectation-density function by introducing more variability. Total rates, however, yielded a different picture. Amphetamine reduced them in a dose-related fashion by introducing extended pausing. Pentobarbital, up to a dose of $2 \mathrm{mg} / \mathrm{kg}$, increased total rates in all 3 monkeys.

Fixed-ratio performance, similar to the ARG in its production of short, uniform IRTs, underwent somewhat corresponding changes. Amphetamine enhanced the similarity of consecutive IRTs, as did the lower doses of pentobarbital. To try to account for such possible results by, say, rate-dependency calculations would compound the perplexity. ARG performance, which intrinsically is high-rate behavior, would be predicted to decline. The close control exercised by the ARG contingency, moreover, turns it into a rather different situation-one in which IRT precision is directly coupled to consequence. Whether this property can account for the performance is not as important as the way in which microanalysis and microcontrol are used to pursue such questions. For example, Weiss and Laties (1964b) reported that performance on a schedule termed stochastic reinforcement of waiting (SRW), which specified reinforcement probability as a linear function of IRT length, also showed a narrowing of IRT distribution in monkeys treated with $0.25 \mathrm{mg} / \mathrm{kg} d, l$-amphetamine.

\section{Markov}

Sequential relationships may also appear or be built into spatial, as well as temporal properties of behavior. Figure 9 depicts a scheme designed to study spatial dependencies. The actual experimental setting consisted of an operant chamber containing three levers. The numerical entries in Figure 9 denote reinforcement probabilities. These values are based on sequences of two responses. For example, the probability of reinforcement for a response on Lever 3 depends on whether the previous response was made on Lever 1, Lever 2, or Lever 3. The highest probability is associated with a previous response on Lever 2 . The name of this reinforcement schedule is taken from the stochastic process in which the transition to a particular state depends only on the previous state, and not on the history of the process prior to that state.

The experimental subjects in this experiment (Weiss \& Heller, 1969) were squirrel monkeys (Saimiri sciurea). Reinforcements consisted of small sucrose pellets. 


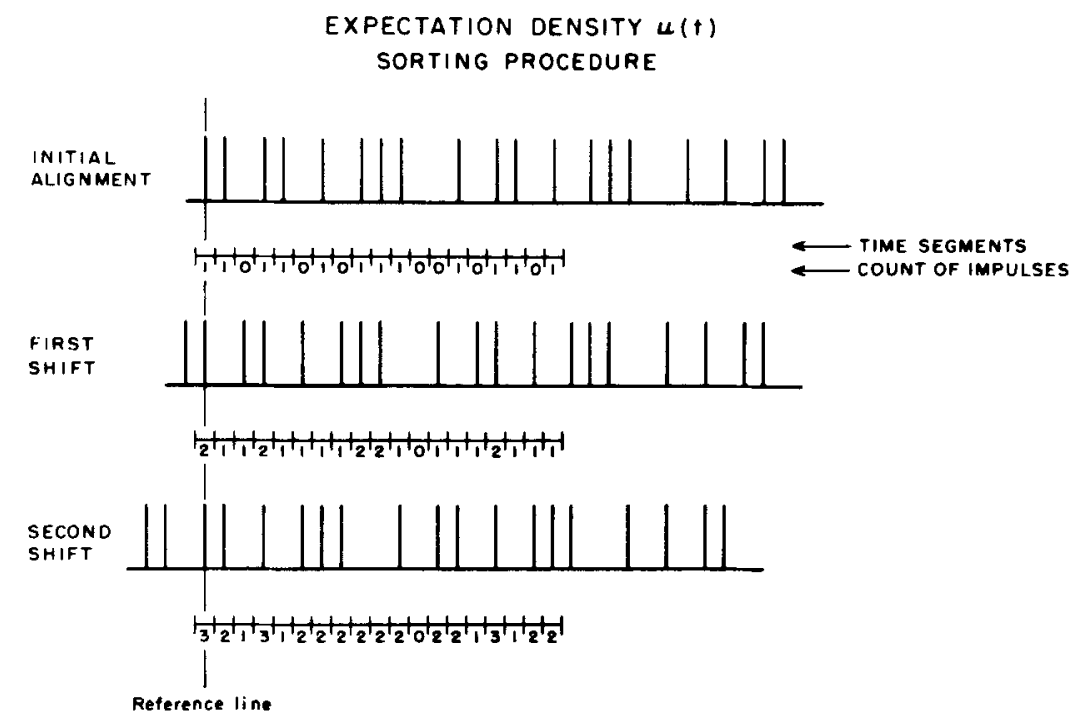

Figure 7. Calculation of expectation-density function. The process basically is a serial sorting procedure. Successive response positions are shifted left and counts added to each histogram (bin $=$ position in time) in which a response appears.

Figure 10 displays both control performance and performance after various doses of an experimental anticholinergic hallucinogen (a phenyl glycolate). The left portion of the figure shows the relative frequencies of sequences of three responses, and the right portion the relative frequencies of sequences of two responses. The values in the right portion are derived from those in the left, since this is a continuous process. Note first that, under control conditions (saline), the relative frequency of possible sequences remained rather stable from session to ses- sion. The sequence 2-3-1, among those beginning with a response on Lever 2, remains most frequent, in accordance with its higher total probability of reinforcement. After drug administration, especially at the two higher doses of 0.6 and $1.2 \mathrm{mg} / \mathrm{kg}$, the distribution shifted in a consistent manner. For example, the sequence 3-1-3, among those defined by a starting point on Lever 3, consistently exceeded in frequency the more optimal sequence 3-1-2. Such consistency suggests a state-dependent process.

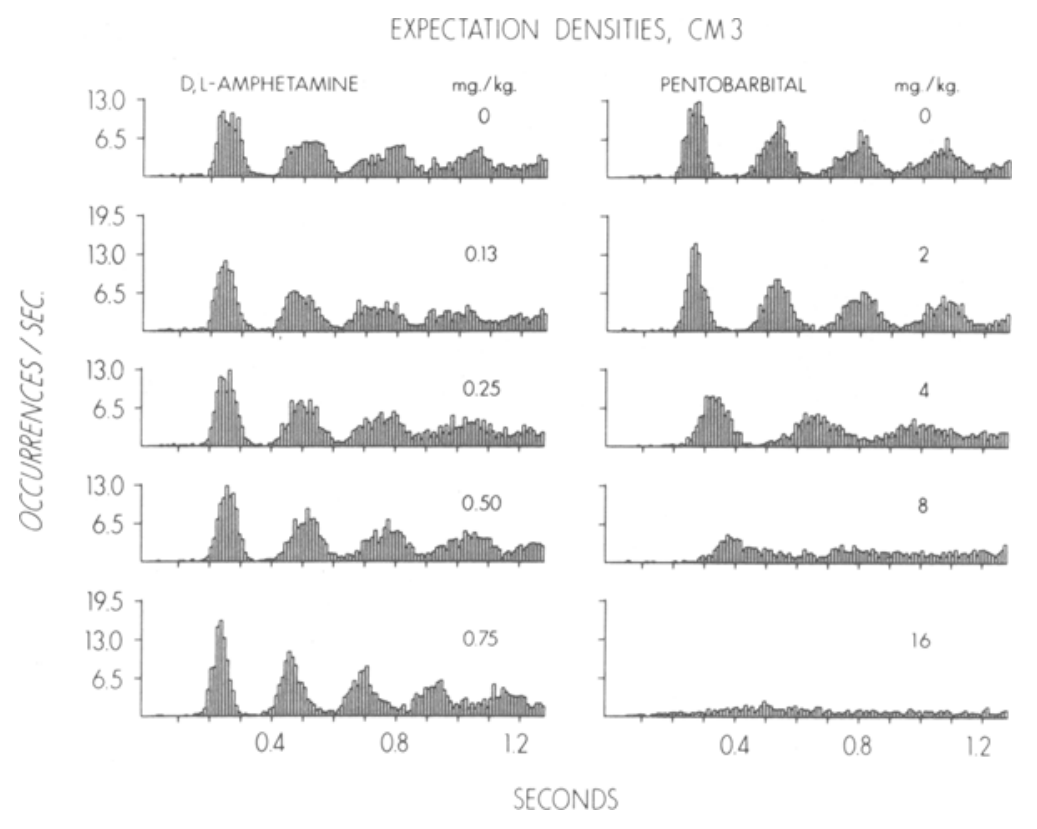

Figure 8. Expectation-density plots showing the effects of $d, l$-amphetamine and pentobarbital on responding maintained by the ARG reinforcement schedule. 


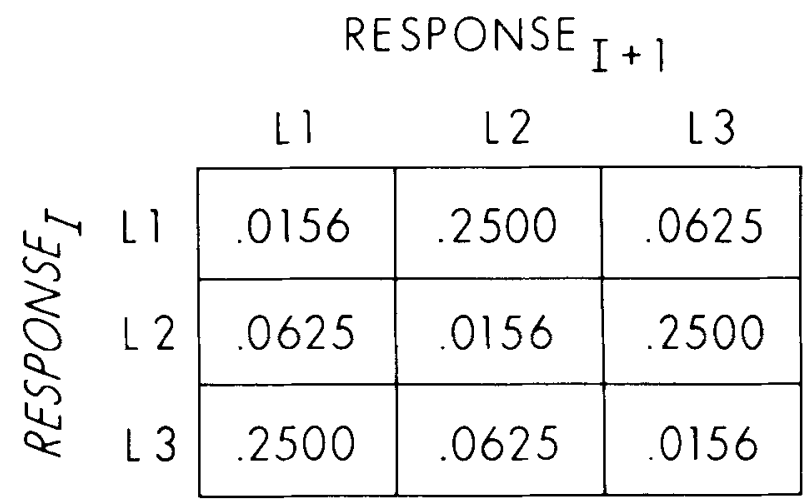

Figure 9. Response matrix depicting probability of reinforcement for response ${ }_{r+1}$ on a particular lever, given the lever position for response,

The Markov schedule resembles the schemes investigated by Thompson (e.g., 1973) and others to determine the modification of learning by drugs. These schemes require subjects to emit fixed sequences of responses. Tracing the acquisition of such response chains is a difficult problem, because unsuccessful sequences cannot easily be defined by degree of correctness. The stochastic properties of the Markov schedule, however, permit the acquisition process to be studied as a gradual shift in sequential choice when the transition probabilities are altered, and the process itself is less encumbered by the problem of sharply reduced reinforcement density.

\section{Shape}

The shape schedule evolved from one designed earlier (Weiss \& Laties, 1964a) to study response duration as a variable influenced by drugs. That earlier study inquired about the way in which dogs would respond to a requirement that they accumulate a specified duration of keypressing (using their snouts) to earn reinforcement. The cumulative response duration schedule showed that the dogs tended to accumulate the required duration (1 $\mathrm{min}$ ) in relatively small increments whose lengths shortened after the administration of amphetamine, pentobarbital, and, especially, combinations of the two. Once online computer control became possible, the scheme was altered to try to lengthen response duration during an experimental session.

The computer program controlling this procedure was based on the premise that the duration criterion should increase gradually if the behavior was not to be lost. When the dog pressed the panel with its snout, the program began to increment the duration timer. If the dog ended the response at a time beyond the required duration, food was delivered and a new criterion calculated. The new criterion was calculated by adding to the previous criterion one fourth of the time by which the response had exceeded the old criterion. This maneuver was designed to prevent an excessively rapid rise in the criterion and the threat of extinction under such circumstances. If the response ended prematurely, a counter was incremented that monitored such responses; if four such durations had occurred in sequence, their mean was calculated, and it served as a term in a calculation by which the criterion was reduced. This process also helped to preserve the behavior. With highly experienced dogs, it was not necessary to lower the criterion; their performance followed the rising criterion with remarkable fidelity.

Amphetamine sharply lowered response durations on this schedule, and, at the same time, it reduced the slope of the rise in the criterion even at doses of $0.1 \mathrm{mg} / \mathrm{kg}$ (Weiss,

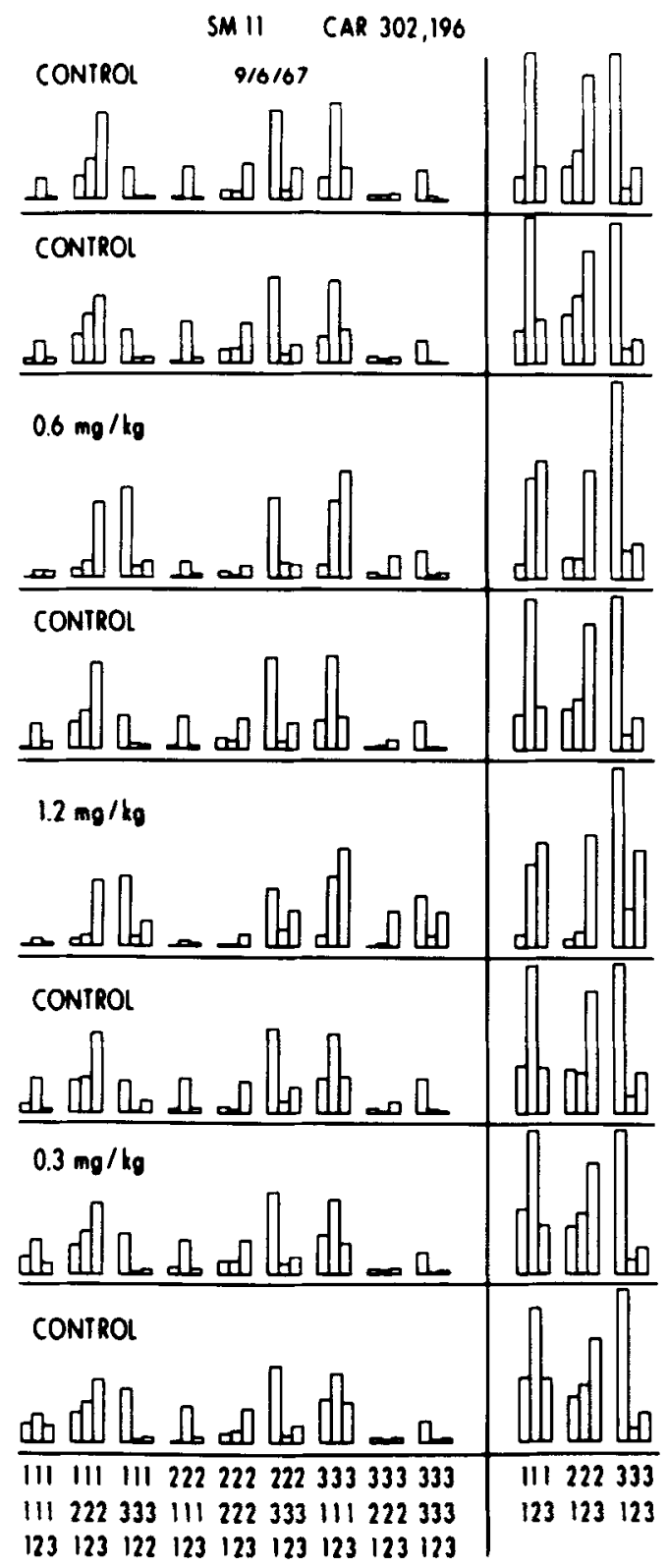

Figure 10. Response patterning for sequences of three (left panel) and sequences of two (right panel) responses on the schedule. Height of bar reflects relative proportion of sequences. Control days yielded different sequential patterns than drug-treatment days. 


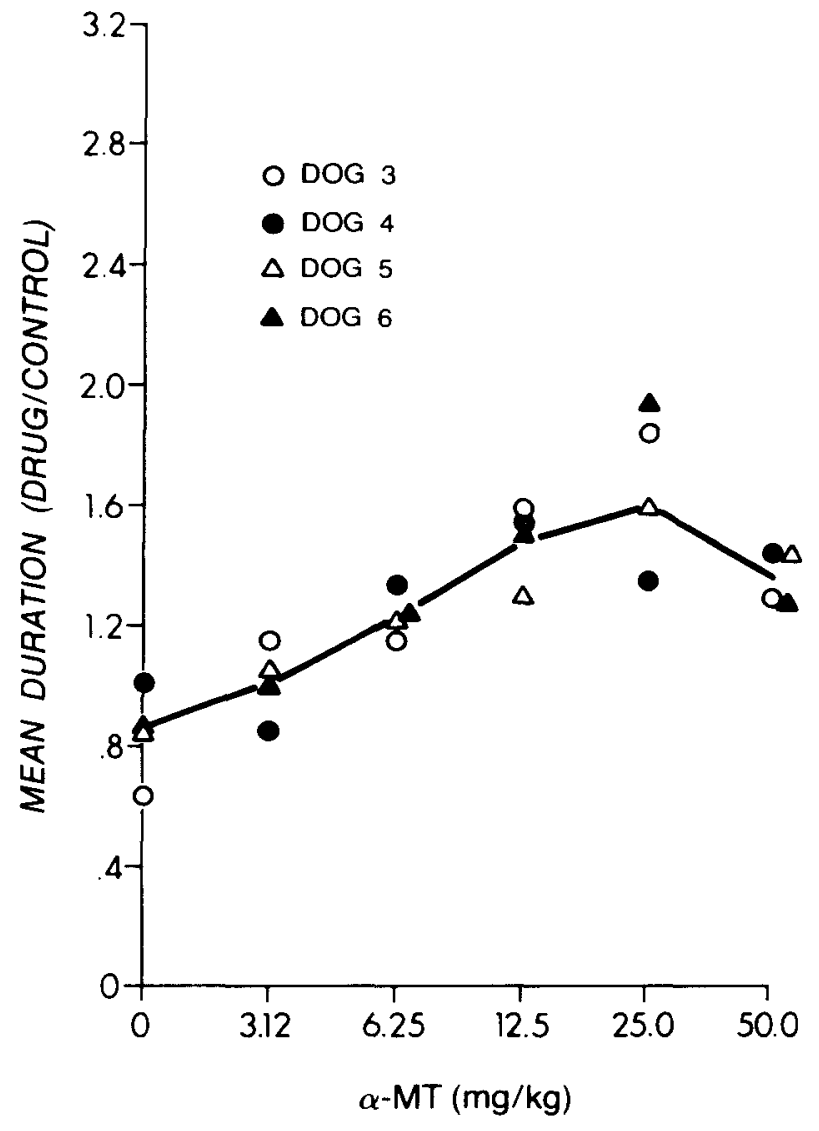

Figure 11. Performance of 4 dogs on schedule after oral administration of $\alpha$-methylparatyrosine. In this version of the schedule, duration criterion could fall.

1970a). An entirely opposite effect was achieved by oral administration of the compound alpha-methylparatyrosine $(\alpha-\mathrm{MT})$, which acts as an inhibitor of the enzyme tyrosine hydroxylase, the rate-limiting enzyme in catecholamine synthesis. Figures 11 and 12 display the performance of 6 dogs after administration of the control solution and after varying doses of $\alpha$-MT. Figure 11 is based on 4 dogs' having been maintained on the original schedule and Figure 12 on two dogs' having been maintained on the modified schedule (i.e., with no lowering of the criterion). Both sets of data suggest the same conclusion: namely, that even relatively low doses of $\alpha$-MT-much lower, in fact, than those used conventionally in psychopharmacologysignificantly lengthen response duration. Figure 13 shows this effect even more dramatically. Each segment compares a drug day with its preceding control day. Each response in a session is represented by a bar. The line weaving its way through the bars is the criterion calculated after the response. Note, on both control days, how little this dog managed to exceed the criterion on successful responses. Also note how much more steeply the criterion climbed after $\alpha$-MT. After $12.5 \mathrm{mg} / \mathrm{kg}$, response durations near the end of the session began to exceed $1 \mathrm{~min}$, and rela- tively few responses, especially with this stringent requirement, remained too short for reinforcement. Given the neurochemical action of $\alpha-\mathrm{MT}$, and the presumed catecholaminergic substrate for amphetamine action, the contrasting performances on the shape schedule produced by these two compounds is intriguing.

\section{DISCUSSION}

Neglecting serial relationships hinders behavioral pharmacology and toxicology just as much as it hinders the experimental analysis of behavior. It means dismissing a crucial variable. For if sequential dependencies exist, then behavioral events initiated by the organism itselfresponses, that is-must be accorded the same status as events generated by the experimenter. Since in almost every situation in which they have been evaluated, serial relationships have emerged, it also seems inescapable that the single response considered in isolation is an abstraction. It is puzzling that almost 50 years since The Behavior

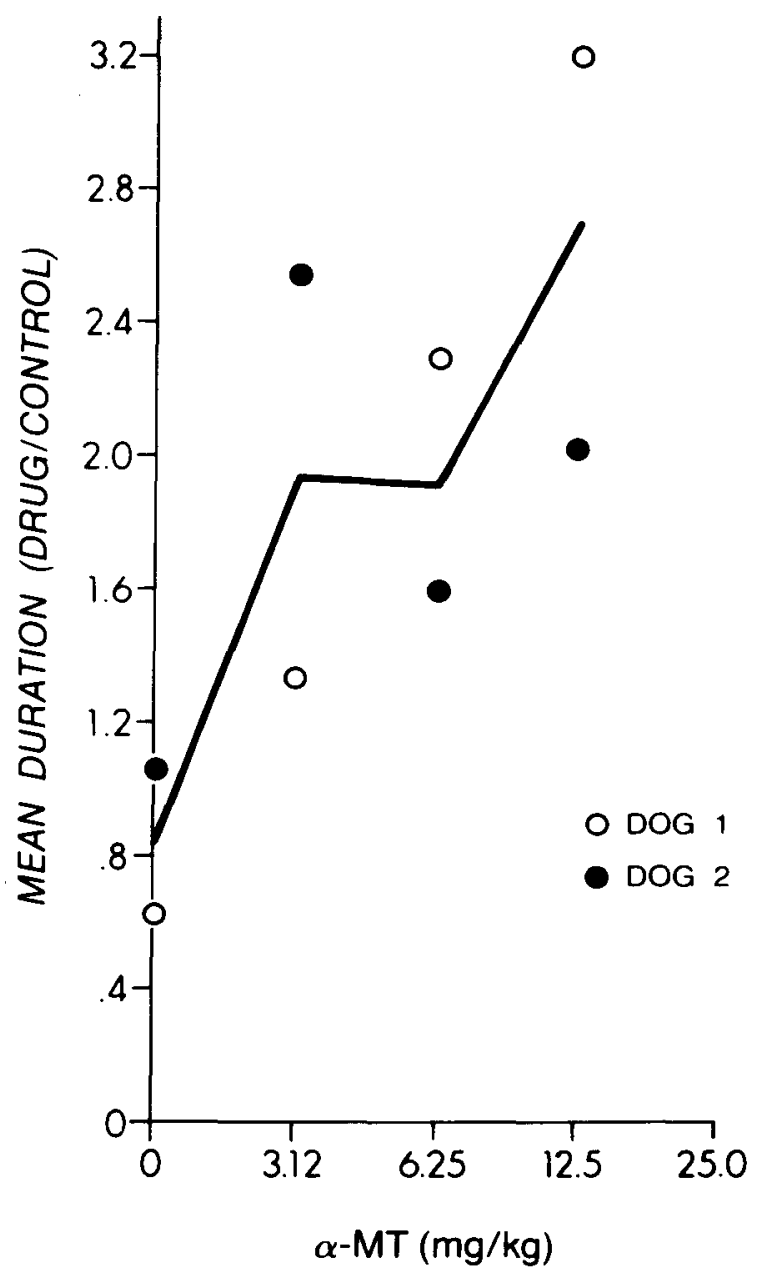

Figure 12. Performance of 2 dogs on schedule after oral administration of $\alpha$-methylparatyrosine. On this version of the schedule, duration criterion could not be decreased but could rise. 


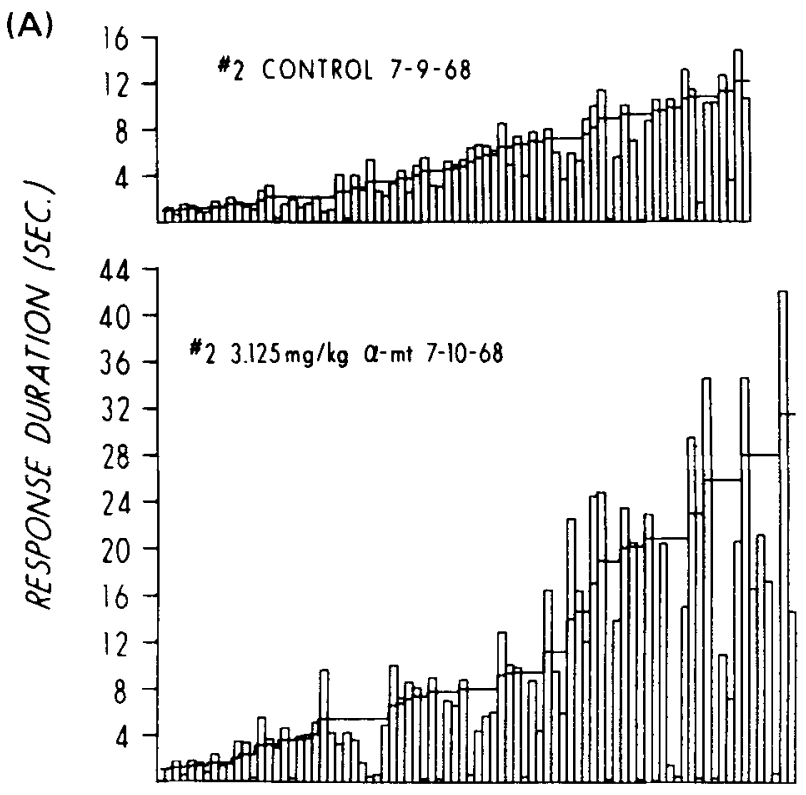

(B)
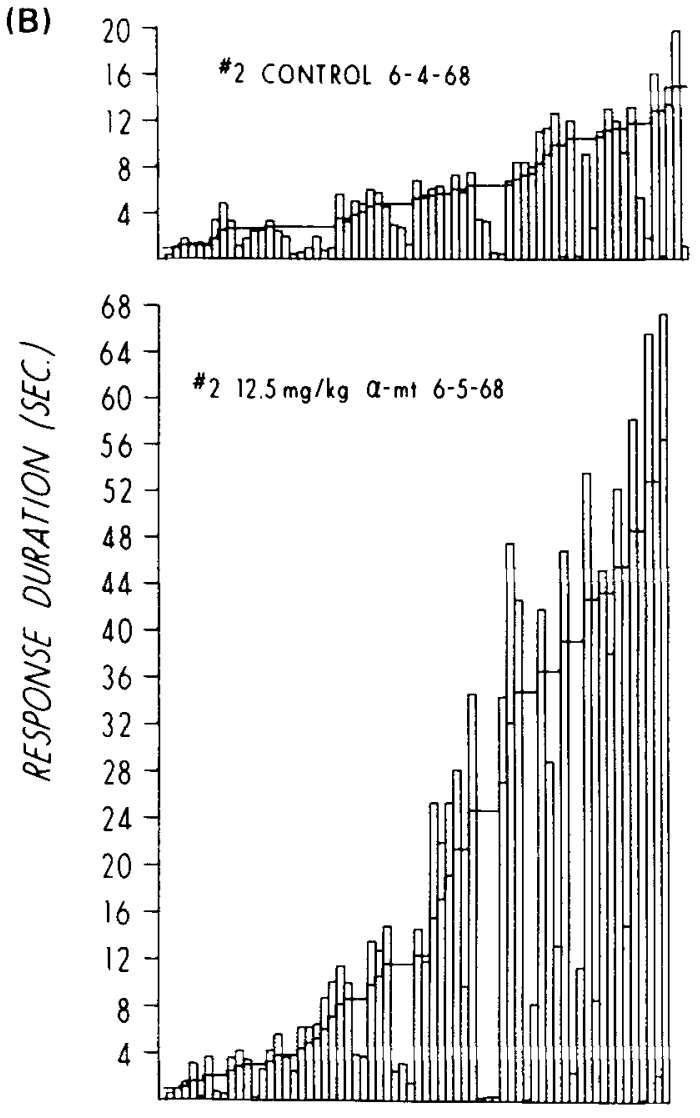

Figure 13. Successive response durations after administration of the control solution and after oral administration of $\alpha$-methylparathyrosine. The line passing through the bars represents the duration criterion. In this version of the schedule, the criterion was not lowered. (A) $3.125 \mathrm{mg} / \mathrm{kg}$, (B) $12.5 \mathrm{mg} / \mathrm{kg}$. of Organisms (Skinner, 1938), so many experimenters persist in ignoring the shaping function of reinforcement (as contrasted with its strengthening function, a distinction offered by Shimp, 1982). What, after all, is the essential difference between shaping a pattern in time and shaping response force or location?

Serial relationships, whether inadvertent or specified, reflect the central elements of behavioral processes based on learning. It does not matter whether experimenters speak of "memory" and "remembering" or use more neutral terms and paradigms. Serial relationships weave themselves through all behavior. For example, they permeate the dispute about the variables underlying choice. That is, does "matching" depend upon some longterm integration of reinforcement frequency (the molar view) or is it governed by more immediately prevailing contingencies (the molecular view)? Many of the arguments, it seems to us, arise out of a lack of information about the contribution of serial relationships.

The same kinds of interpretive ambiguity arise with drug actions on behavior, largely because so much of the literature of behavioral pharmacology is based on gross measures of rate. Rate is useful in many ways, and ratedependency computations can be revealing with regard to the importance of establishing the baseline conditions from which inferences are drawn; but they convey little about the process. Response probability, in fact, was the crucial datum that Skinner sought for the experimental analysis of behavior, and the cumulative record became its pictorial representation. The sequential patterning described by "elbows," "knees," "break-and-run," and other terms have been displaced by abstract mathematical formulations that draw further and further away from the behavior itself. Microanalysis, in many respects, is an amplification of the cumulative record, reaching beyond it to restructure and even to recreate it.

Experimenters and theorists alike cannot afford to ignore how easily the conventional reinforcement schedules discussed in this paper yield complex serial relationships, some of which determine many of the effects on rate, while others reveal distinct details of the pharmacological effects. Those schedules designed to exploit serial relationships reveal other aspects of how these relationships respond to chemical challenge. The published data, however, remain too sparse even to support speculation about the mechanisms responsible for the observed effects. The problem is that serial relationships in behavior have largely been ignored, even now, when the technology for studying them is so richly available.

Serial relationships are only one aspect of a more universal problem, however. Both experimenters and theorists rely mostly on crude molar data, such as overall rates, to describe what are essentially molecular processes. They are probably misled by the ease with which molar contingencies can be formulated and implemented. For example, the verbal description of a fixed-interval rein- 
forcement schedule reveals nothing of the complex performance it maintains. Increasingly elaborate models simply distance the theorist from the actual molecular contingencies.

The conclusions of this chapter can be stated succinctly. The microstructure of behavior is not random. If we are to understand the details of how chemicals modify behavior, it will not suffice to rely on gross molar measures. Chemicals alter the response of organisms to environmental events and conditions in many ways. To reject the microanalysis of behavior is to direct almost all responsibility for any observed effects to states within the organism itself, and, in essence, to deny that the outcomes arise from the complex interactions of chemical and environmental variables.

\section{REFERENCES}

Branch, M. N., Gollub, L. R. (1974). A detailed analysis of the effects of $d$-amphetamine on behavior under fixed-interval schedules. Journal of the Experimental Analysis of Behavior, 21, 519-539.

Ferster, C. B., SkINNER, B. F. (1957). Schedules of reinforcement. New York: Appleton-Century-Crofts.

GAGE, M. I. (1970). Amphetamine and pentobarbital effects on interresponse time distributions of monkeys reinforced for low sequential variability of interresponse times. Unpublished doctoral dissertation, University of Rochester, Rochester, NY.

Gentry, D. G., Weiss, B., \& LATIES, V. G. (1983). The microanalysis of fixed-interval responding. Journal of the Experimental Analysis of Behavior, 39, 327-343.

Gotr, T. C., \& WeIss, B. (1972). The development of fixed-ratio performance under the influence of ribonucleic acid. Joumal of the Experimental Analysis of Behavior, 18, 481-497.

Laties, V. G., Weiss, B., Weiss, A. B. (1969). Further observations on overt "mediating"' behavior and the discrimination of time. Journal of the Experimental Analysis of Behavior, 12, 43-57.

MAZUR, J. E. (1982). A molecular approach to ratio schedule performance. In M. L. Commons, R. J. Herrnstein, \& H. Rachlin (Eds.), Quantitative analyses of behavior: Vol II. Matching and maximizing accounts (pp. 79-110). Cambridge, MA: Ballinger.

Millenson, J. R., HuRWitz, H. M. B. (1961). Some temporal and sequential properties of behavior during conditioning and extinction. Journal of the Experimental Analysis of Behavior, 4, 97-106.

Miller, G. A., FRICK, F. C. (1949). Statistical behavioristics and sequences of responses. Psychological Review, 56, 311-324.

Morse, W. H., \& HerRnstein, R. J. (1956). Effects of drugs on characteristics of behavior maintained by complex schedules of intermittent positive reinforcement. Annals of the New York Academy of Sciences, 65, 303-317.

Morse, W. H., \&e Keleher, R. T. (1970). Schedules as fundamental determinants of behavior. In W. N. Schoenfeld \& J. Farmer (Eds.), The theory of reinforcement schedules (pp. 139-185). New York: Appleton-Century-Crofts.

PogGio, G. F., VIERnstein, L. J. (1964). Time series analysis of impulse sequences of thalamic somatic sensory neurons. Journal of Neurophysiology, 27, 517-545.

SchWARTZ, B. (1980). Development of complex stereotyped behavior in pigeons. Journal of the Experimental Analysis of Behavior, 33, 153-166.

SHIMP, C. P. (1973). Sequential dependencies in free-responding. Journal of the Experimental Analysis of Behavior, 19, 491-497.
Shimp, C. P. (1981). Local structure of steady-state operant behavior. In C. M. Bradshaw, E. Szabadi, \& C. F. Lowe (Eds.), Quantification of steady-state operant behavior (pp. 189-203). Amsterdam: Elsevier.

SHIMP, C. B. (1982). Reinforcement and the local organization of behavior. In M. L. Commons, R. J. Herrnstein, \& H. Rachlin (Eds.), Quantitative analyses of behavior: Vol. II. Matching and maximizing accounts (pp. 111-130). Cambridge, MA: Ballinger.

Silberberg, A., Hamilton, B., Ziriax, J. M., Casey, J. (1978). The structure of choice. Joumal of Experimental Psychology: Animal Behavior Processes, 4, 368-398.

SilberberG, A., Ziriax, J. M. (1982). The interchangeover time as a molecular dependent variable in concurrent schedules. In $\mathbf{M}$. $\mathbf{L}$. Commons, R. J. Herrnstein, \& H. Rachlin (Eds.), Quantitative analyses of behavior: Vol. II. Matching and maximizing accounts (pp. 131151). Cambridge, MA: Ballinger.

SKInNer, B. F. (1938). The behavior of organisms. New York: Appleton-Century-Crofts.

Thompson, D. M. (1973). Repeated acquisition as a behavioral baseline for studying drug effects. Joumal of Pharmacology \& Experimental Therapeutics, 184, 506-514.

Weiss, B. (1970a). Amphetamine and the temporal structure of behavior. In E. Costa \& S. Garrattini (Eds.), International symposium on amphetamines and related compounds (pp. 797-817). New York: Raven Press.

WEISs, B. (1970b). The fine structure of operant behavior during transition states. In W. N. Schoenfeld \& J. Farmer (Eds.), The theory of reinforcement schedules (pp. 277-311). New York: AppletonCentury-Crofts.

WEISs, B. (1973a). Can computers answer behavioral questions? Behavior Research Methods \& Instrumentation, 5, 67-79.

WeIss, B. (1973b). Digital computers and the microanalyses of behavior. In B. Weiss (Ed.), Digital computers in the behavioral laboratory (pp. 99-140). New York: Appleton-Century-Crofts.

WEISs, B. (1981). Microproperties of operant behavior as aspects of toxicity. In C. M. Bradshaw, E. Sbabadi, \& C. E. Lowe (Eds.), Quantification of steady-state operant behavior (pp. 249-265). Amsterdam: Elsevier.

WEISs, B., GoTT, T. C. (1972). A microanalysis of drug effects on fixed-ratio performance in pigeons. Journal of Pharmacology \& Experimental Therapeutics, 180, 189-202.

WeISs, B., HeLler, A. (1969). Methodological problems in evaluating the role of cholinergic mechanisms in behavior. Federation Proceedings, 28, 135-146.

Weiss, B., \& LATIES, V. G. (1964a). Effects of amphetamine, chlorpromazine, pentobarbital, and ethanol on operant response duration. Journal of Pharmacology \& Experimental Therapeutics, 144, 17-23.

Weiss, B., LATIES, V. G. (1964b). Drug effects on the temporal patterning of behavior. Federation Proceedings, 23, 801-807.

WEISS, B., LATIES, V. G. (1965). Reinforcement schedule generated by an on-line digital computer. Science, 148, 658-661.

Weiss, B., LAtres, V. G. (1967). Comparative pharmacology of drugs affecting behavior. Federation Proceedings, 26, 1146-1156.

Weiss, B., Laties, V. G., Siegel, L., \& Goldstein, D. (1966). A computer analysis of serial interactions in spaced responding. Journal of the Experimental Analysis of Behavior, 9, 619-625.

WiLliams, D. R. (1968). The structure of response rate. Journal of the Experimental Analysis of Behavior, 11, 251-258.

(Manuscript received March 10, 1986; revision accepted for publication June 17, 1987.) 